\title{
Peripheral Arterial Disease in Patients Presenting with Acute Coronary Syndrome in Six Middle Eastern Countries
}

\author{
Hassan A. Al-Thani, ${ }^{1}$ Ayman El-Menyar, ${ }^{2,3}$ Mohammad Zubaid, ${ }^{4}$ \\ Wafa A. Rashed, ${ }^{5}$ Mustafa Ridha, ${ }^{6}$ Wael Almahmeed, ${ }^{7}$ Kadhim Sulaiman, ${ }^{8}$ \\ Ahmed Al-Motarreb, ${ }^{9}$ Haitham Amin, ${ }^{10}$ and Jassim Al Suwaidi ${ }^{2,3}$ \\ ${ }^{1}$ Department of Vascular Surgery, Hamad General Hospital, P.O. Box 3050, Doha, Qatar \\ ${ }^{2}$ Department of Cardiology and Cardiovascular Surgery, Hamad General Hospital, P.O. Box 3050, Doha, Qatar \\ ${ }^{3}$ Clinical Medicine, Weill Cornell Medical School, P.O. Box 24144, Doha, Qatar \\ ${ }^{4}$ Department of Medicine, Faculty of Medicine, Kuwait University, P.O. Box 24923, Kuwait \\ ${ }^{5}$ Department of Medicine, Mubarak Al-Kabeer Hospital, P.O. Box 43787, Ministry of Health, Kuwait \\ ${ }^{6}$ Division of Cardiology, Department of Medicine, Adan Hospital, P.O. Box 46969, Ministry of Health, Kuwait \\ ${ }^{7}$ Sheikh Khalifa Medical City, P.O. Box 51900, Abu Dhabi, UAE \\ ${ }^{8}$ Royal Hospital, P.O. Box 1331, Muscat, Oman \\ ${ }^{9}$ Department of Medicine, Faculty of Medicine, Sana'a University, Sana'a, Yemen \\ ${ }^{10}$ Mohammed Bin Khalifa Cardiac Centre, Manama, Bahrain
}

Correspondence should be addressed to Hassan A. Al-Thani, althanih@hotmail.com

Received 31 May 2011; Accepted 21 September 2011

Academic Editor: Karlheinz Peter

Copyright (C) 2011 Hassan A. Al-Thani et al. This is an open access article distributed under the Creative Commons Attribution License, which permits unrestricted use, distribution, and reproduction in any medium, provided the original work is properly cited.

To describe prevalence and impact of peripheral arterial disease (PAD) in patients with acute coronary syndrome (ACS), data were collected over 5 months from 6 Middle Eastern countries. Patients were divided into 2 groups (with and without PAD). Out of 6705 consecutive ACS patients, PAD was reported in 177 patients. In comparison to non-PAD, PAD patients were older and more likely to have cardiovascular risk factors. They were more likely to have high Killip class, high GRACE risk score, and non-ST elevation ACS (NSTEACS) at presentation. Thrombolytics, antiplatelet use, and coronary intervention were comparable in both groups. When presented with ST-elevation myocardial infarction (STEMI), patients with PAD had worse outcomes, while in NSTEACS; PAD was associated with higher rate of heart failure in comparison to non-PAD patients. In diabetics, PAD was associated with 2 -fold increase in mortality when compared to non-PAD $(P=0.028)$. After adjustment, PAD was associated with high mortality in STEMI (adjusted OR 2.6; 95\% CI 1.23-5.65, $P=0.01$ ). Prevalence of PAD in ACS in the Gulf region is low. Patients with PAD and ACS constitute a high risk group and require more attention. PAD in patients with STEMI is an independent predictor of in-hospital death.

\section{Introduction}

The prevalence of peripheral arterial disease (PAD) is variable and relatively high in the western world [1-4]. Patients with PAD are at increased risk of coronary, carotid and cerebrovascular atherosclerosis disease, and all-cause mortality [5-8]. This risk is independent of the traditional risk factors such as diabetes mellitus, hypertension, smoking, and obesity [8-10]. PAD is not a static disease and its progression from intermittent claudication to rest pain or gangrene can occur [7-10]. It is possible that the functional impairment in patients with PAD may keep them from ambulating to the point of having angina to the extent that those patients may present with much more advanced coronary atherosclerosis [5]. This risk becomes greater as the severity of PAD increases $[7,8]$. Several studies have shown worse prognosis in acute coronary syndrome (ACS) when PAD present in both selected and unselected western population admitted with ACS $[1,3,5,11-15]$. However, the prevalence and the impact of PAD in patients with 
acute coronary syndrome in the Middle Eastern countries are limited. The aim of the current study is to study the prevalence of the PAD and to evaluate its impact on the inhospital mortality and major adverse cardiac events across the ACS population in the Middle Eastern population.

\section{Methods}

For the purpose of the current analysis, data for 6705 consecutive ACS patients was collected from a 6-month prospective, multicenter study of the Gulf Registry of Acute Coronary Events (Gulf RACE) from 6 adjacent Middle Eastern Gulf countries (Bahrain, Kuwait, Qatar, Oman, United Arab Emirates, and Yemen). Patients were recruited from 64 hospitals with the diagnosis of ACS including unstable angina (UA) and non-ST- and ST-elevation myocardial infarction (NSTEMI and STEMI). There were no exclusion criteria and thus all the prospective patients with ACS were actually enrolled. The study received ethical approval from the institutional ethical bodies in all participating countries. Full details of the methods have been published $[16,17]$. Data were collected on record forms by the treating physicians. Completed data sheets were sent to the central data processing center, for uniform monitoring and registration. We analyzed patients with peripheral arterial disease (PAD) compared them with those who did not have PAD.

2.1. Definitions. Briefly, diagnosis of the different types of ACS and definitions of data variables were based on the American College of Cardiology clinical data standard [18]. For the purpose of this report, ST-segment elevation myocardial infarction and left bundle branch block myocardial infarction were grouped together and called STEMI, whereas merging NSTEMI and unstable angina patients called NSTEACS.

2.2. Peripheral Arterial Disease. In addition to welldocumented previous history of PAD (i.e., vascular surgery or angioplasty), ankle-brachial index (ABI) of $<0.8$ in either leg was used as cut point for the presence of PAD. To calculate the ABI ratio, the average systolic blood pressure measurement in the ankle was divided by the average systolic blood pressure measurement in the arm. The mean pressure of the higher arm was used to calculate the ABI separately for each leg.

2.3. Statistical Analysis. Patients were divided into 2 groups (with and without PAD). Clinical and biochemical variables, comorbidities and in-hospital medical treatment in ACS patients were analyzed in both groups. Data were presented as proportion or mean \pm standard deviation $(\mathrm{SD})$ as appropriate. Differences in categorical variables between respective comparison groups were analyzed using the $\chi^{2}$ test. The continuous variables were analyzed using independent-samples $t$-test. The primary end points were analyzed and compared in the different groups using the $\chi^{2}$ test. Multivariate logistic regression analysis was carried out after controlling for the relevant variables for the predictors of hospital outcomes. Primary end points included in-hospital reischemia, heart failure (HF), and mortality. The multivariate analysis was adjusted for following potential covariates: age, sex, diabetes mellitus, hypertension, aspirin, heparin, glycoprotein inhibitors, and coronary angiography. Adjusted odds ratios, with accompanying 95\% confidence intervals, were reported for the respective categories. Moreover, multivariate logistic regression analysis was carried out after controlling for the relevant variables for the predictors of PAD. All $P$ values were two-sided tailed. $P$ values of $<0.05$ were considered significant. All data analyses were carried out using the Statistical Package for Social Sciences version 18 (SPSS Inc. USA). For the purpose of comparing our findings with the western experiences, we selected 4 western originated studies that were conducted on PAD patients presenting with ACS. These studies were SPRINT (Secondary Prevention Study Reinfarction Israeli Nifedipine Trial), GRACE (Global Registry of Acute Coronary Events), PAMISCA (Prevalencia de Afectación de Miembros Inferiores en el paciente con Síndrome Coronario Agudo), and MASCARA (Manejo del Sindrome Coronario Agudo. Registro Actualizado) $[1,11,14$, $15]$.

\section{Results}

3.1. Clinical and Biochemical Profiles. Out of the 6705 patients who were admitted with ACS, PAD was documented in 177 patients (2.6\%). Table 1 shows the baseline characteristics and risk factors of patients with PAD in comparison to non-PAD patients. Patients with PAD were 9 years older $(65 \pm 11$ versus $56 \pm 12, P<0.0001)$, and were more likely to be female ( $35 \%$ versus $24 \%, P<0.001)$. PAD patients were also more likely to have diabetes mellitus (69\% versus $40 \%, P<0.001)$, hypertension $(77 \%$ versus $50 \%, P<0.001)$, dyslipidemia (66\% versus $31 \%, P=0.001$ ), previous history of CAD (79\% versus $45 \%, P<0.001)$, prior coronary revascularization $(35 \%$ versus $15 \%, P<0.001)$, chronic lung disease $(17.5 \%$ versus $5 \%)$ and renal failure $(45 \%$ versus $17 \%, P=0.001)$. They were less likely to be smokers $(32 \%$ versus $38 \%, P=0.001)$. At presentation with ACS, PAD patients had higher heart rate, Killip class, and GRACE risk score $(P<0.001$ for all). Mean total cholesterol was lesser in PAD group $(4.6 \pm 1.5$ versus $5.02 \pm 2.3, P=0.001)$ and mean serum triglyceride value was comparable in the 2 groups. NSTEACS was the most frequent diagnosis in PAD patients, whereas STEMI was the predominant diagnosis in non-PAD group.

3.2. In-Hospital Treatment Pattern. Table 2 demonstrates the treatment patterns for patients with and without PAD. In regard to on admission therapy, there were no differences between the two groups in the use of oral and intravenous antiplatelet medications, thrombolysis therapy, angiotensinconverting enzyme, or angiotensinogen-receptor inhibitors use. Coronary interventions were also comparable in the 2 groups. In PAD group, unfractionated heparin and $\beta$-blocker were used less frequently $(P=0.001)$, while low molecular 
TABLE 1: Clinical and biochemical profiles of patients with acute coronary syndrome.

\begin{tabular}{|c|c|c|c|}
\hline & Non-PAD $(N=6528)$ & $\operatorname{PAD}(N=177)$ & $P$ value \\
\hline Age (mean) & $56 \pm 12$ & $65 \pm 11$ & 0.001 \\
\hline Females (\%) & $1571(24)$ & $61(35)$ & $<.001$ \\
\hline Prior coronary artery disease (\%) & $2929(45)$ & $140(79)$ & $<.001$ \\
\hline Prior coronary revascularization (\%) & $987(15)$ & $62(35)$ & $<.001$ \\
\hline Family history of CAD (\%) & $873(13)$ & $29(16)$ & 0.87 \\
\hline Diabetes mellitus (\%) & $2622(40)$ & $123(70)$ & $<.001$ \\
\hline Hypertension & $3228(50)$ & $136(77)$ & $<.001$ \\
\hline Smoking (\%) & $2491(38)$ & $56(32)$ & $<.001$ \\
\hline Dyslipidemia (\%) & $2034(31)$ & $116(66)$ & $<.001$ \\
\hline Prior aspirin use $(\%)$ & $2644(41)$ & $141(80)$ & $<.001$ \\
\hline Renal failure (\%) & $1091(17)$ & $80(45)$ & $<.001$ \\
\hline Chronic lung disease (\%) & $335(5)$ & $31(17.5)$ & $<.001$ \\
\hline Heart rate (mean, b/min) & $86 \pm 22$ & $93 \pm 27$ & $<.001$ \\
\hline SBP (mean, mmgh) & $140 \pm 30$ & $137 \pm 33$ & 0.26 \\
\hline DBP (mean, mmgh) & $84 \pm 17$ & $78 \pm 18$ & 0.001 \\
\hline BMI (mean) & $27.6 \pm 5$ & $27.4 \pm 5$ & 0.77 \\
\hline Ischemic chest pain, $n(\%)$ & $5223(80)$ & $121(68)$ & $<.001$ \\
\hline Late presentation & $801(31)$ & $13(28)$ & 0.61 \\
\hline Killip class >I, $n(\%)$ & $1392(21)$ & $79(45)$ & $<.001$ \\
\hline LV ejection fraction $<40 \%(\%)$ & $890(22)$ & $40(33)$ & 0.007 \\
\hline NSTEACS & $3957(61)$ & $129(73)$ & 0.001 \\
\hline STEMI/LBBB & 2571(39) & $48(27)$ & 0.001 \\
\hline \multicolumn{4}{|l|}{ GRACE risk Scoring } \\
\hline Low, $n(\%)$ & $2047(43)$ & $14(11)$ & \\
\hline Medium, $n(\%)$ & $1341(28)$ & $22(18)$ & $<.001$ \\
\hline High, $n(\%)$ & $1390(29)$ & $90(71)$ & \\
\hline \multicolumn{4}{|l|}{ Biochemical findings } \\
\hline First blood sugar (mg/dL) & $11 \pm 10$ & $13 \pm 7$ & $<.001$ \\
\hline Fasting blood sugar (mg/dL) & $8 \pm 8$ & $9 \pm 4$ & 0.02 \\
\hline Peak troponin (ng/mL) & $17 \pm 51$ & $7 \pm 30$ & 0.04 \\
\hline First creatinine $(\mu \mathrm{mol} / \mathrm{L})$ & $107 \pm 92$ & $163 \pm 139$ & $<.001$ \\
\hline Total cholesterol & $5 \pm 2$ & $4.6 \pm 1.5$ & $<.001$ \\
\hline $\mathrm{HDL}(\mu \mathrm{mol} / \mathrm{L})$ & $1.03 \pm 1.2$ & $0.97 \pm 0.7$ & 0.22 \\
\hline $\mathrm{LDL}(\mu \mathrm{mol} / \mathrm{L})$ & $3.3 \pm 3$ & $4.6 \pm 2$ & 0.001 \\
\hline Fasting triglyceride $(\mu \mathrm{mol} / \mathrm{L})$ & $1.9 \pm 2$ & $1.8 \pm 1.2$ & 0.77 \\
\hline First haemoglobin (mean, gm/L) & $14 \pm 4$ & $13 \pm 6$ & 0.009 \\
\hline
\end{tabular}

PCI: Percutaneous coronary interventions, CAD: coronary artery disease, SBP: systolic blood pressure, DBP: diastolic blood pressure. GRACE: Global registry of acute coronary events.

weight heparin was more frequently used $(P=0.03)$. At discharge, aspirin and $\beta$-blocker were less likely used $(P=$ $0.001)$, while clopidogrel and statin were more likely used in PAD group ( $P=0.03$ and 0.01 resp.).

3.3. In-Hospital Clinical Outcomes. Table 3 shows hospital outcomes in overall, STEMI and NSTEACS patients. In overall ACS and STEMI, all the primary end points were significantly worse in PAD group in addition to the higher percent of bleedings and stroke. In NSTEACS patients, there were no significant differences between the groups except for the high percent of the incidence of heart failure in PAD group (24\% versus $16 \%, P=0.009)$. Hospital stay was significantly prolonged in PAD group in overall (6.2 versus 5.6 days, $P=0.03$ ) and NSTEACS (6.4 versus 5.2 days, $P=0.003$ ) patients. In diabetic patients, PAD was associated with 2-fold increase in mortality when compared to nonPAD ( $8 \%$ versus $4 \%, P=0.028$ ) (Figure 1 ). There was a significant main effect for $\operatorname{PAD}(P=0.008)$ and not for DM $(P=0.63)$ on the mortality; there was no interaction between the two variables $(P$ interaction $=0.98)$.

3.3.1. Multivariate Logistic Regression Analysis (Figure 2). After adjustment for the important variables (traditional risk factors), the independent predictors for PAD were DM (OR1.9; 95\% CI 1.36-2.77), renal failure (OR 2.5; 
TABLE 2: Management of patients with acute coronary syndrome.

\begin{tabular}{lccc}
\hline & No-PAD & PAD & $P$ value \\
\hline On admission medications & & & \\
Thrombolysis, $n(\%)$ & $1494(58)$ & $24(51)$ & 0.34 \\
Aspirin, $n(\%)$ & $6391(98)$ & $172(97)$ & 0.49 \\
Clopidogrel, $n(\%)$ & $3499(54)$ & $106(60)$ & 0.09 \\
Heparin, $n(\%)$ & $3093(48)$ & $67(38)$ & 0.01 \\
LMW Heparin, $n(\%)$ & $3073(47)$ & $98(55)$ & 0.03 \\
Gp IIb/IIIa inhibitor, $n(\%)$ & $677(10)$ & $23(13)$ & 0.26 \\
$\beta$-Blocker, $n(\%)$ & $4277(66)$ & $84(48)$ & 0.001 \\
ACE/ARB, $n(\%)$ & $4498(69)$ & $120(68)$ & 0.74 \\
Coronary angiography (\%) & $1217(19)$ & $33(19)$ & 0.99 \\
PCI, $n(\%)$ & $273(19.14 .2)$ & $7(19.94)$ & 0.88 \\
Discharge medications & & & \\
Aspirin, $n(\%)$ & $6155(95)$ & $150(85)$ & 0.001 \\
Clopidogrel, $n(\%)$ & $3204(49)$ & $101(57)$ & 0.03 \\
Statin, $n(\%)$ & $5264(81)$ & $156(88)$ & 0.01 \\
ACE/ARB, $n(\%)$ & $4950(76)$ & $132(75)$ & 0.66 \\
$\beta$-Blocker, $n(\%)$ & $4939(76)$ & $107(61)$ & 0.001 \\
Diuretics, $n(\%)$ & $4561(70)$ & $134(76)$ & 0.11 \\
\hline
\end{tabular}

TABle 3: Clinical outcomes in patients with acute coronary syndromes.

\begin{tabular}{lccc}
\hline & Non-PAD & PAD & $P$ value \\
\hline Overall & & & \\
In-hospital death, $n(\%)$ & $233(4)$ & $14(8)$ & 0.002 \\
Heart failure, $n(\%)$ & $1044(16)$ & $55(31)$ & 0.001 \\
Recurrent ischemia, $n$ (\%) & $580(10)$ & $24(14)$ & 0.03 \\
Re-infarction, $n(\%)$ & $151(2.3)$ & $2(1.1)$ & 0.29 \\
Major bleeding, $n(\%)$ & $46(0.7)$ & $6(3.4)$ & 0.001 \\
Stroke, $n$ (\%) & $45(0.7)$ & $4(2.3)$ & 0.01 \\
Hospital stay (mean) (days) & $5.6 \pm 4.6$ & $6.2 \pm 4.2$ & 0.03 \\
STEMI/LBBB & & & \\
In-hospital death, $n$ (\%) & $161(6)$ & $11(23)$ & 0.001 \\
Heart failure, $n(\%)$ & $430(17)$ & $24(50)$ & 0.001 \\
Recurrent ischemia, $n$ (\%) & $233(9)$ & $9(19)$ & .02 \\
Major bleeding, $n(\%)$ & $26(1)$ & $5(10)$ & 0.001 \\
Stroke, $n$ (\%) & $29(1.1)$ & $3(6.4)$ & 0.001 \\
Hospital stay (mean) (days) & $6.14 \pm 4.3$ & $5.8 \pm 3.7$ & 0.58 \\
NSTEACS & & & \\
In-hospital death, $n$ (\%) & $72(1.8)$ & $3(2.3)$ & 0.67 \\
Heart failure, $n(\%)$ & $614(16)$ & $31(24)$ & 0.009 \\
Recurrent ischemia, $n$ (\%) & $347(9)$ & $15(12)$ & 0.27 \\
Major bleeding, $n(\%)$ & $20(0.5)$ & $1(0.8)$ & 0.67 \\
Stroke, $n$ (\%) & $16(0.4)$ & $1(0.8)$ & 0.52 \\
Hospital stay (mean) (days) & $5.17 \pm 4.8$ & $6.4 \pm 4.4$ & 0.003 \\
\hline
\end{tabular}

95\% CI 11.79-3.43), smoking (OR 1.8; 95\% CI 1.23-2.63), prior CAD (OR 2.3; 95\% CI 1.49-3.38), and dyslipidemia (OR 2.3; 95\% CI 1.54-3.22). For in-hospital mortality, after adjusting for relevant variables including age, sex, risk factors, treatment, and coronary angiography, PAD was

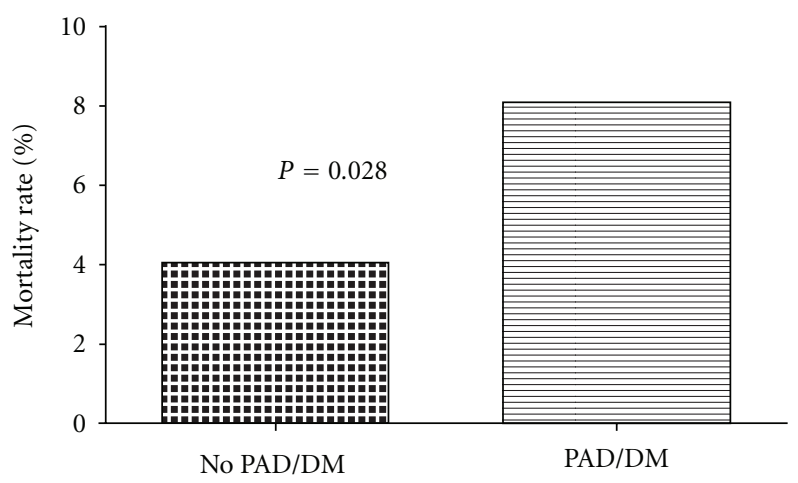

(a)

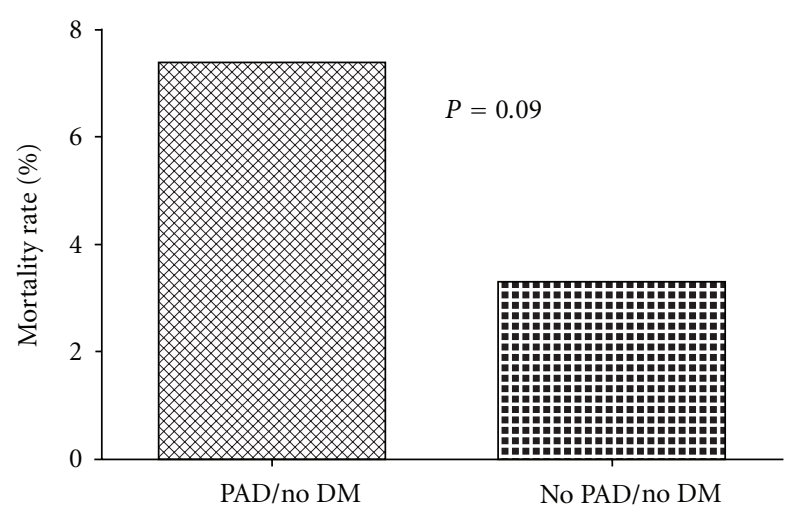

(b)

FIgURE 1: Mortality rate in peripheral arterial disease (PAD) patients presenting with acute coronary syndrome patients and stratified by the diabetic status (DM).

an independent predictor of mortality in STEMI patients (adjusted OR 2.6; 95\% CI 1.23-5.65, $P=0.01$ ).

\section{Discussion}

The current study demonstrated the prevalence and impact of PAD among patients presenting with ACS who are living in the Middle East. There are several key findings in the present study. Firstly, the prevalence of PAD among ACS patients is low in the gulf region in comparison to the western populations $[1,3,11-15]$. This finding may indicate the underestimation or missed PAD diagnosis in the initial evaluation of ACS patients. Secondly, PAD is a marker of worse baseline cardiovascular risk profile. In overall ACS and STEMI, patients with PAD developed worse in-hospital outcomes in terms of greater rate of death, heart failure, recurrent ischemia, stroke, and major bleeding when compared to their non-PAD counterparts. This was consistent with the previous western studies [1, 11, 14, 15]. Even after adjusting for the potential relevant covariants, PAD was an independent predictor for mortality in STEMI patients in the current study. PAD increased the rate of death almost 3 times in comparison to non-PAD patients. In NSTEACS patients, PAD was associated with significant higher rate of heart failure in comparison to non-PAD. This 
Predictors of peripheral arterial disease

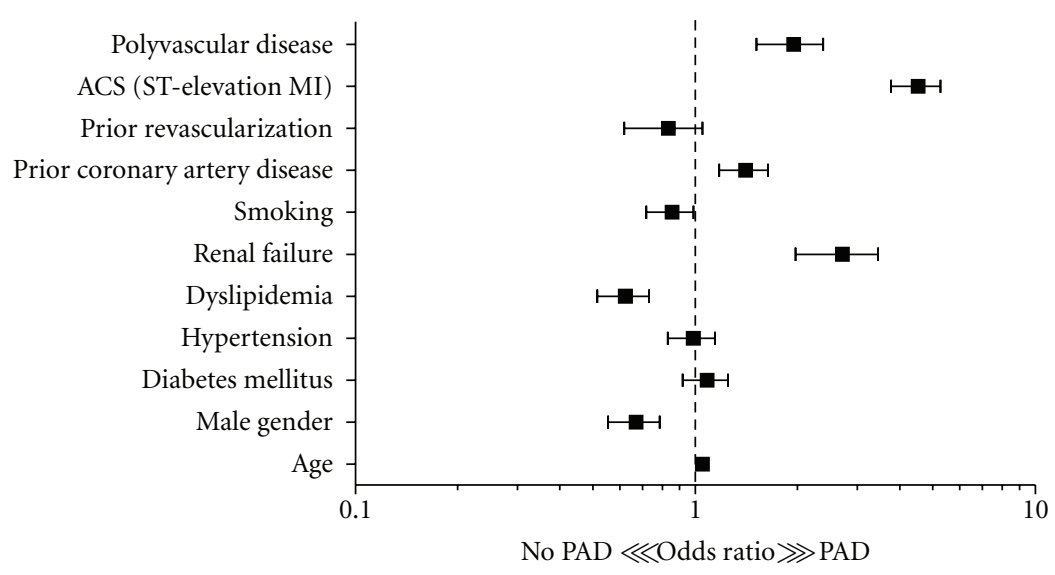

(a)

Predictors of in-hospital mortality in ST-elevation myocardial infarction patients

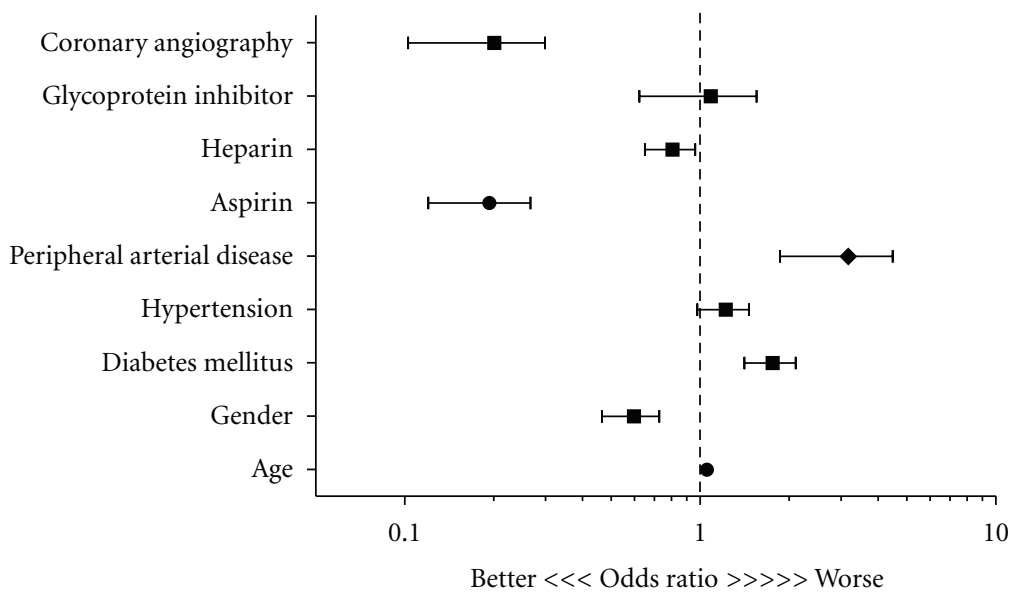

(b)

FIGURE 2: (a) predictors for peripheral arterial disease, (b) clinical predictors for in-hospital mortality in ST-elevation myocardial infarction.

was consistent with the data from the CRUSADE registry in which PAD was an independent predictor of HF in NSTACS patients [5]. Thirdly, in diabetic patients, PAD increased in-hospital mortality rate twice when compared to their counterpart non-PAD patients. In a recent study, Lafitte et al. [19] reported 3-fold increase in the risk of cardiovascular $(\mathrm{CV})$ events in patients with both PAD and diabetes even after optimization of risk-factor control and medications. This long-term high CV risk was not significant in diabetic patients without PAD.

Previous studies reported that although PAD patients were high risk group, they were less likely to be appropriately treated with evidence-based therapy and this may in part explain the worse outcomes $[3,4,12,13]$. However, in the current study, there were no significant differences in the management between the 2 groups apart from the fewer use of $\beta$ blockers in PAD group. $\beta$ blockers were not frequently used because of the presence of PAD per se and high percent of chronic lung disease in the PAD group. Fourthly,
PAD patients ranked high GRACE risk scoring in patients presenting with ACS; this might be useful simple bedside tool for early risk-stratification of those patients.

Many traditional and emergent CV risk factors are more prevalent in patients with ACS and PAD. Also, certain factors are independent predictors of PAD [10]. However, the associated increased $\mathrm{CV}$ risk is independent of those traditional risk factors. The main independent predictors for PAD in the present study included DM, renal failure, prior CAD, and dyslipidemia. The prevalence of PAD in patients with ACS has been reported in several studies in the range of $1 \%$ to $39.8 \%$ [20-22]. This wide range is influenced by the same factors as the patients without ACS. The prevalence of PAD among patients with CAD varies between studies according to the method of diagnosis [2]. The PAD prevalence ranged between 2.4 in the current study to 39.8 in the PAMISCA study (Table 4). The prevalence in the PAMISCA study was higher than all the other studies and the authors attributed this to the high subclinical PAD patients and the exclusion 


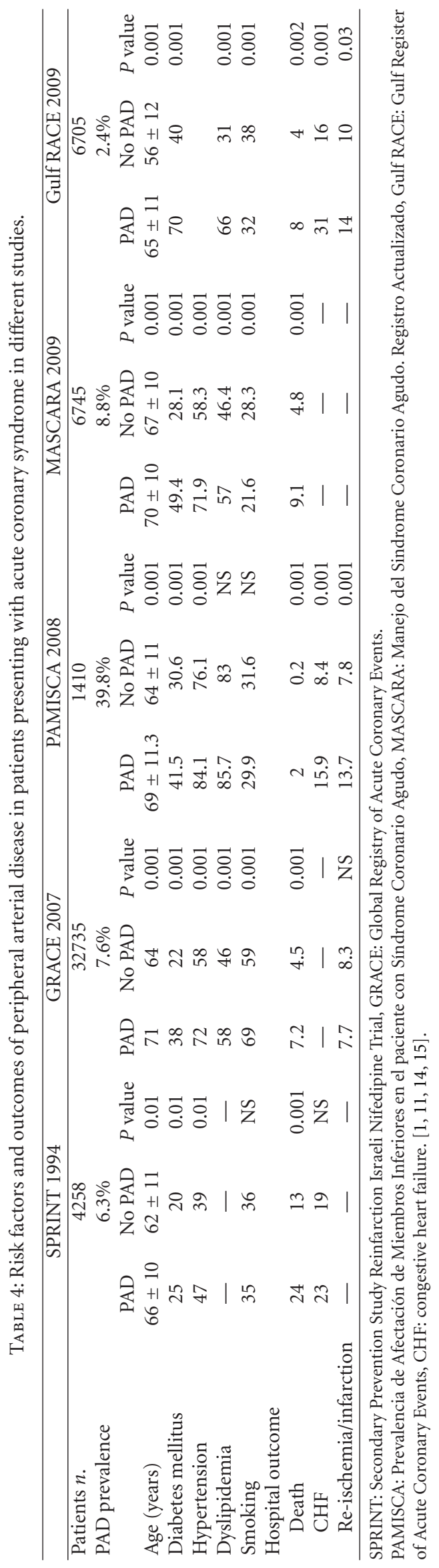


of patients younger than 40 years of age. In our study, the presence of PAD is probably underestimated in part due to the inclusion criteria $(\mathrm{ABI}$ of $<0.8)$, the high prevalence of diabetes mellitus (70\%) which can lead to a false elevation of the ankle pressure, and slightly younger age group. The present study reported the highest prevalence of diabetes mellitus when compared to other studies which ranged from $25 \%$ to $49.4 \%$. Hypertension and dyslipidemia were higher in patients with PAD when compared with non-PAD patients in all the studies. On the other hand, smoking was lower in patient with PAD compared to non-PAD patients in all the studies with the exception of GRACE study. There were fewer smokers in PAD group in the present study, but interestingly, in multivariate logistic regression analysis, smoking became one of the independent predictors for PAD. Recently, Conen et al. [23] assessed the association of smoking status with PAD in healthy women. The investigators reported that smoking was a potent risk factor for symptomatic PAD and was associated with subclinical inflammation. Also, they observed that smoking cessation substantially reduced risk for PAD, but an increased occurrence of PAD persisted even among former smokers [23].

Data on the prevalence and outcome of Middle Eastern patients with PAD are very limited. Al Zahrani et al. [24] in a cross-sectional hospital-based study (402 patients) in Saudi Arabia reported high prevalence of PAD $(\mathrm{ABI}<0.9)$ in elderly high risk patients with either diabetes mellitus (61.4\%), chronic renal failure $(13.4 \%)$, or ischemic heart disease (21.4\%) when compared to controls (4.1\%). Previously, we conducted a cross-sectional multicenter study in 5 Middle Eastern countries [25]. The study enrolled 1,341 patients who were either (1) with cardiovascular disease (cerebrovascular, ischemic heart disease, and/or peripheral vascular disease) or (2) were at risk of developing cardiovascular disease based on the presence of cardiovascular risk factors. At that study, we reported high prevalence of PAD based on $\mathrm{ABI}<0.9$ (31.5\% and $28.2 \%$, resp.). The current study extended these observations and reported for the first time, in our region, the prognostic impact of PAD among ACS patients.

Our data were collected from an observational study which is one of the limitations. However, well-designed observational studies provide valid results and do not systematically overestimate the results compared with the results of randomized controlled trials. Moreover, inconsistent cutoff points for ABI would potentially miss patients with milder PAD leading to potential outcome bias in different studies.

\section{Conclusion}

Prevalence of PAD in ACS in the Gulf region is low. Patients with PAD and ACS are high risk group that require more attention for risk factors and early detection. Certain traditional risk factors are independent predictors for PAD necessitates aggressive preventive measures. PAD in patients with STEMI is an independent predictor for inhospital death. Detection of PAD in ACS patients might be a useful simple bedside tool for early detection of the risk stratification.

\section{Funding}

Gulf RACE is a Gulf Heart Association project and was financially supported by Sanofi Aventis, Paris, France and Qatar Telecommunications Company, Doha, Qatar. The sponsors had no role in study design, data collection, or data analysis. The sponsors had no role in the writing of the paper and its submission.

\section{Conflict of Interests}

The authors have no conflict of interests.

\section{Acknowledgment}

The authors would like to thank all the staff in all the participating centers for their cooperation.

\section{References}

[1] S. Behar, M. Zion, H. Reicher-Reiss, E. Kaplinsky, and U. Goldbourt, "Short- and long-term prognosis of patients with a first acute myocardial infarction with concomitant peripheral vascular disease," American Journal of Medicine, vol. 96, no. 1, pp. 15-19, 1994.

[2] S. Kownator, J. P. Cambou, P. Cacoub et al., "Prevalence of unknown peripheral arterial disease in patients with coronary artery disease: data in primary care from the IPSILON study," Archives of Cardiovascular Diseases, vol. 102, no. 8-9, pp. 625631, 2009.

[3] A. Meizels, D. M. Zeitoun, V. Bataille et al., "Impact of polyvascular disease on baseline characteristics, management and mortality in acute myocardial infarction. The Alliance project," Archives of Cardiovascular Diseases, vol. 103, no. 4, pp. 207-214, 2010.

[4] J. J. F. Belch, E. J. Topol, G. Agnelli et al., "Prevention of atherothrombotic disease network. Critical issues in peripheral arterial disease detection and management: a call to action," Archives of Internal Medicine, vol. 163, no. 8, pp. 884892, 2003.

[5] D. L. Bhatt, E. D. Peterson, R. A. Harrington et al., "Prior polyvascular disease: risk factor for adverse ischaemic outcomes in acute coronary syndromes," European Heart Journal, vol. 30, no. 10, pp. 1195-1202, 2009.

[6] P. Sabouret, P. Cacoub, J. Dallongeville et al., "REACH: international prospective observational registry in patients at risk of atherothrombotic events. Results for the French arm at baseline and one year," Archives of Cardiovascular Diseases, vol. 101, no. 2, pp. 81-88, 2008.

[7] M. H. Criqui, J. K. Ninomiya, D. L. Wingard, M. Ji, and A. Fronek, "Progression of peripheral arterial disease predicts cardiovascular disease morbidity and mortality," Journal of the American College of Cardiology, vol. 52, no. 21, pp. 1736-1742, 2008.

[8] A. M. Shah, T. Banerjee, and D. Mukherjee, "Coronary, peripheral and cerebrovascular disease: a complex relationship," Journal of the Indian Medical Association, vol. 108, no. 5, pp. 292-296, 2010. 
[9] A. B. Newman, L. Shemanski, and T. A. Manolio, "Anklearm index as a predictor of cardiovascular disease and mortality in the cardiovascular health study: the cardiovascular health study group," Arteriosclerosis, Thrombosis, and Vascular Biology, vol. 19, pp. 538-545, 1999.

[10] A. Huelmos, J. Jiménez, C. Guijarro et al., "Underrecognized peripheral arterial disease in patients with acute coronary syndrome: prevalence of traditional and emergent cardiovascular risk factors," Revista Espanola de Cardiologia, vol. 58, no. 12, pp. 1403-1410, 2005.

[11] I. Ferreira-González, G. P. Miralda, M. Heras et al., "Prognosis and management of patients with acute coronary syndrome and polyvascular disease," Revista Espanola de Cardiologia, vol. 62, no. 9, pp. 1012-1021, 2009.

[12] J. L. Januzzi, J. Buros, and C. P. Cannon, "Peripheral arterial disease, acute coronary syndromes, and early invasive management: the TACTICS TIMI 18 trial," Clinical Cardiology, vol. 28, no. 5, pp. 238-242, 2005.

[13] G. Cotter, C. P. Cannon, C. H. McCabe et al., "Prior peripheral arterial disease and cerebrovascular disease are independent predictors of adverse outcome in patients with acute coronary syndromes: are we doing enough? Results from the orbofiban in patients with unstable coronary syndromes-thrombolysis in myocardial infarction (OPUS-TIMI) 16 study," American Heart Journal, vol. 145, no. 4, pp. 622-627, 2003.

[14] D. Mukherjee, K. A. Eagle, E. Kline-Rogers et al., "Impact of prior peripheral arterial disease and stroke on outcomes of acute coronary syndromes and effect of evidence-based therapies (from the global registry of acute coronary events)," American Journal of Cardiology, vol. 100, no. 1, pp. 1-6, 2007.

[15] P. Morillas, J. Quiles, A. Cordero et al., "Impact of clinical and subclinical peripheral arterial disease in mid-term prognosis of patients with acute coronary syndrome," American Journal of Cardiology, vol. 104, no. 11, pp. 1494-1498, 2009.

[16] A. El-Menyar, M. Zubaid, K. Sulaiman et al., "In-hospital major clinical outcomes in patients with chronic renal insufficiency presenting with acute coronary syndrome: data from a registry of 8176 patients," Mayo Clinic Proceedings, vol. 85, no. 4, pp. 332-340, 2010.

[17] A. El-Menyar, M. Zubaid, W. Rashed et al., "Comparison of men and women with acute coronary syndrome in six middle eastern countries," American Journal of Cardiology, vol. 104, no. 8, pp. 1018-1022, 2009.

[18] C. P. Cannon, A. Battler, R. G. Brindis et al., "American college of cardiology key data elements and definitions for measuring the clinical management and outcomes of patients with acute coronary syndromes: a report of the American College of Cardiology task force on clinical data standards (Acute Coronary Syndromes Writing Committee)," Journal of the American College of Cardiology, vol. 38, no. 7, pp. 21142130, 2001.

[19] M. Lafitte, L. Barandon, Y. Pucheu et al., "After acute coronary syndrome, diabetic patients with peripheral vascular disease remain at high risk of cardiovascular events despite secondary prevention measures," Archives of Cardiovascular Diseases, vol. 103, no. 2, pp. 97-105, 2010.

[20] V. Bertomeu, P. Morillas, J. R. Gonzalez-Juanatey et al., "Prevalence of peripheral arterial disease in patients with acute coronary síndrome (PAMISCA) Investigators. Prevalence and prognostic influence of peripheral arterial disease in patients $\geq 40$ years old admitted into hospital following an acute coronary event," European Journal of Vascular and Endovascular Surgery, vol. 36, no. 2, pp. 189-196, 2008.
[21] C. Diehm, S. Kareem, and H. Lawall, "Epidemiology of peripheral arterial disease," Journal of Vascular Diseases, vol. 33, no. 4, pp. 183-189, 2004.

[22] F. G. R. Fowkes, L. P. Low, S. Tuta, and J. Kozak, "Anklebrachial index and extent of atherothrombosis in 8891 patients with or at risk of vascular disease: results of the international AGATHA study," European Heart Journal, vol. 27, no. 15, pp. 1861-1867, 2006.

[23] D. Conen, B. M. Everett, T. Kurth et al., "Smoking, smoking status, and risk for symptomatic peripheral artery disease in women," Annals of Internal Medicine, vol. 154, no. 11, pp. 719726, 2011.

[24] H. A. Al Zahrani, H. M. S. Al Bar, A. Bahnassi, and A. A. Abdulaal, "The distribution of peripheral arterial disease in a defined population of elderly high-risk Saudi patients," International Angiology, vol. 16, no. 2, pp. 123-128, 1997.

[25] A. El-Menyar, H. Amin, I. Rashdan et al., "Ankle-brachial index and extent of atherosclerosis in patients from the middle east (the AGATHA-ME Study): a cross-sectional multicenter study," Angiology, vol. 60, no. 3, pp. 329-334, 2009. 


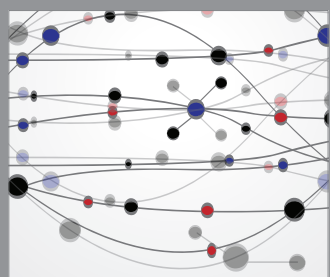

The Scientific World Journal
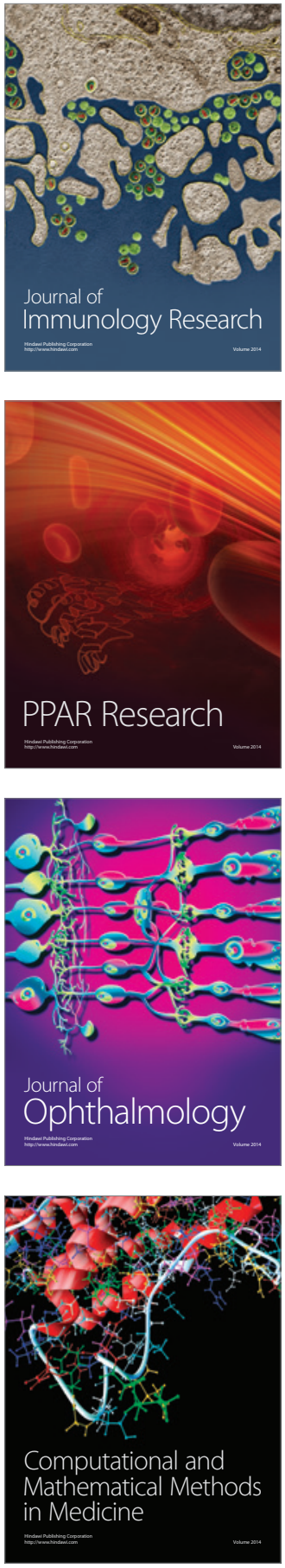

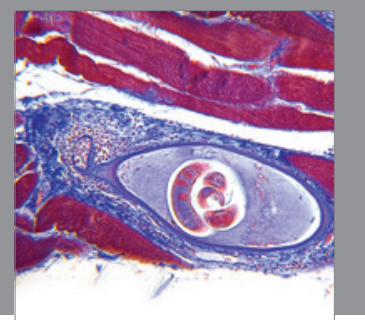

Gastroenterology

Research and Practice
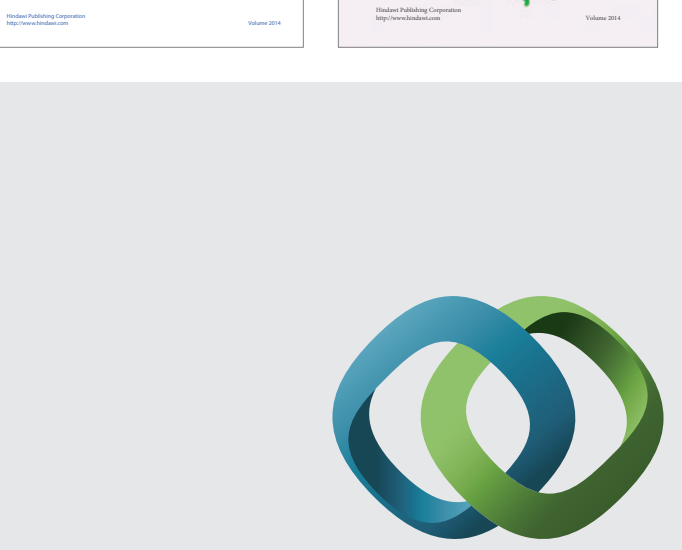

\section{Hindawi}

Submit your manuscripts at

http://www.hindawi.com
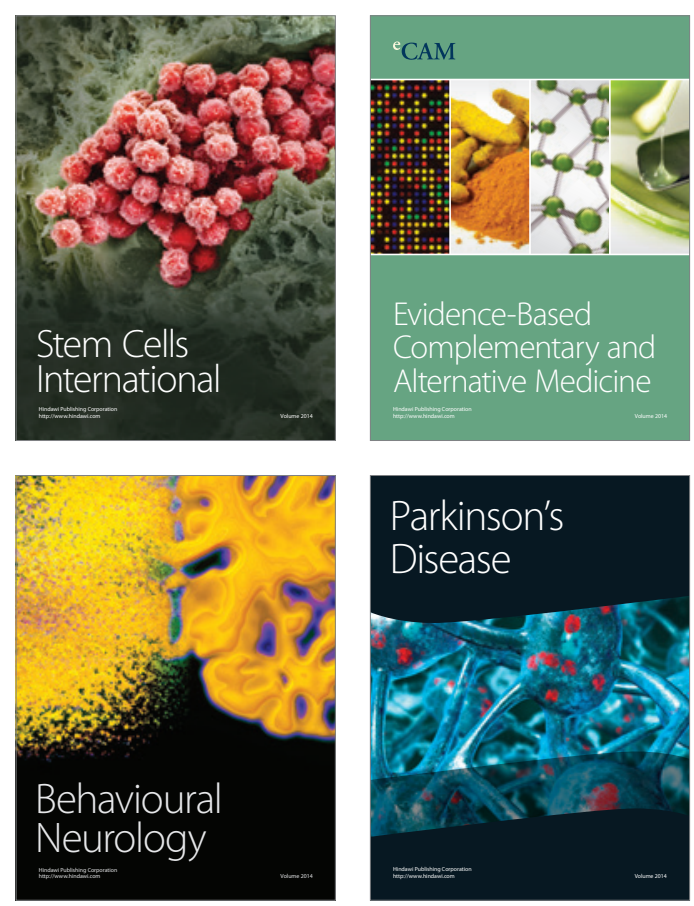

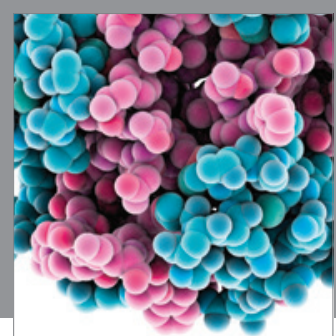

Journal of
Diabetes Research

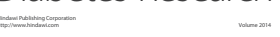

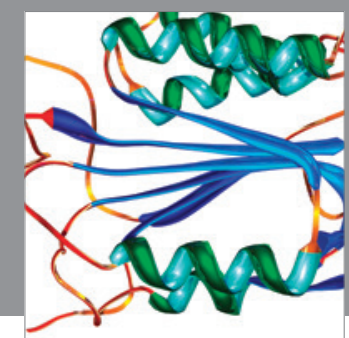

Disease Markers
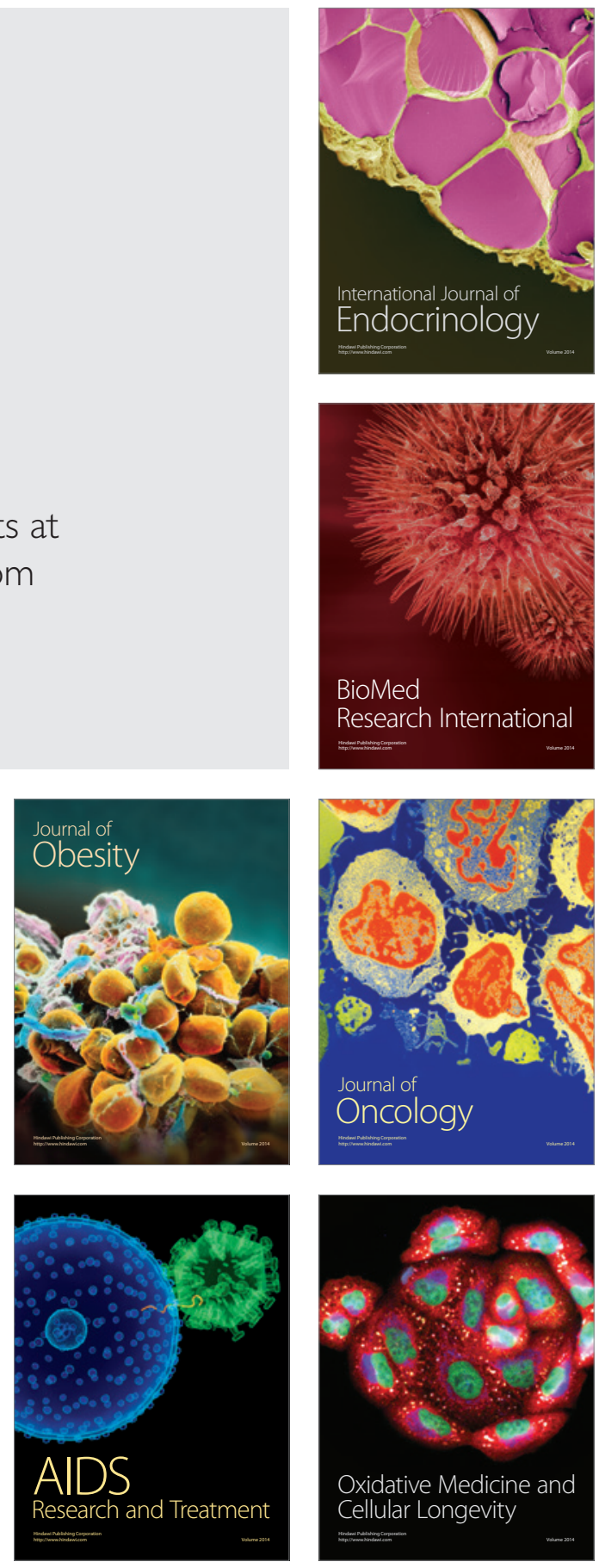